\title{
Discovery and Optimization of Novel Pyridines as Highly Potent and Selective Glycogen Synthase Kinase 3 Inhibitors
}

\author{
Savithri Ramurthy ${ }^{\mathrm{a}}$, Keith B. Pfister ${ }^{\mathrm{b}}$, Rustum S. Boyce ${ }^{\mathrm{c}}$, Sean P. Brown ${ }^{\mathrm{d}}$, Abran Q. \\ Costales ${ }^{\mathrm{e}}$, Manoj Desai ${ }^{\mathrm{f}}$, Eric Fang ${ }^{\mathrm{g}}$, Barry H. Levine ${ }^{\mathrm{h}}$, Simon C. $\mathrm{Ng}^{\mathrm{i}}$, John M. Nuss ${ }^{\mathrm{j}}$, \\ David B. Ring ${ }^{\mathrm{k}}$, Cynthia M. Shafer ${ }^{\mathrm{l}}$, Wei Shu ${ }^{\mathrm{b}}$, Sharadha Subramanian ${ }^{\mathrm{m}}$, Allan S. \\ Wagman $^{\mathrm{n}}$, Haixia Wang ${ }^{\mathrm{o}}$ and Dirksen E. Bussiere ${ }^{\mathrm{b}, *}$

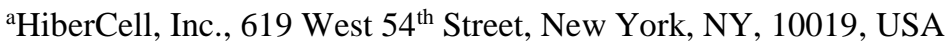 \\ ${ }^{\mathrm{b}}$ Global Discovery Chemistry, Novartis Institutes for BioMedical Research, 5300 Chiron Way, Emeryville, CA, 94608, USA \\ ${ }^{c}$ Vermont Biosynthetics LLC, 365 Glinnis Road, Northfield, VT, 05663, USA \\ ${ }^{d}$ Proneurotech, 161 Oyster Point Blvd., Suite 200, South San Francisco, CA, 94080, USA \\ ${ }^{\mathrm{e}}$ Chevron Oronite Company LLC, 100 Chevron Way, Richmond, CA, 94801, USA \\ ${ }^{\mathrm{f}}$ Gilead Sciences, 333 Lakeside Drive, Foster City, CA, 94404, USA \\ gProtein Sciences, Novartis Institutes for BioMedical Research, 5300 Chiron Way, Emeryville, CA 94608, USA \\ h2026 South Lincoln Avenue, Spokane, WA, 99203, USA \\ i311 Preakness Court, Walnut Creek, CA, 94597, USA \\ jOppilan Pharma, Ltd., 332 Encinitas Blvd., Encinitas, CA, 92024, USA \\ k2375 Cowper Street, Palo Alto, CA, 94301, USA \\ 'BeiGene, 1900 Powell Street, Suite 500, Emeryville, CA. 94608, USA \\ m3305 Cydonia Ct., Dublin, CA. 94568, USA \\ ${ }^{n}$ Rain Therapeutics Inc., 8000 Jarvis Avenue, Suite 204, Newark, CA 94560, USA \\ ${ }^{\circ}$ Takeda San Diego Inc., 10410 Science Center Dr., San Diego, CA, 92121, USA
}

Received October 29, 2019; Accepted Month XX, 2019

\begin{abstract}
Glycogen synthase kinase-3 plays an essential role in multiple biochemical pathways in the cell, particularly in regards to energy regulation. As such, Glycogen synthase kinase-3 is an attractive target for pharmacological intervention in a variety of disease states, particularly non-insulin dependent diabetes mellitus. However, due to homology with other crucial kinases, such as the cyclin-dependent protein kinase CDC2, developing compounds that are both potent and selective is challenging. A novel series of derivatives of 5-nitro-N2-(2-(pyridine-2ylamino)ethyl)pyridine-2,6-diamine were synthesized and have been shown to potently inhibit glycogen synthase kinase-3 (GSK3). Potency in the low nanomolar range was obtained along with remarkable selectivity. The compounds activate glycogen synthase in insulin receptor-expressing CHOIR cells and in primary rat hepatocytes, and have acceptable pharmacokinetics and pharmacodynamics to allow for oral dosing. The X-ray co-crystal structure of human GSK3- $\beta$ in complex with compound $\mathbf{2}$ is reported and provides insights into the structural determinants of the series responsible for its potency and selectivity.
\end{abstract}

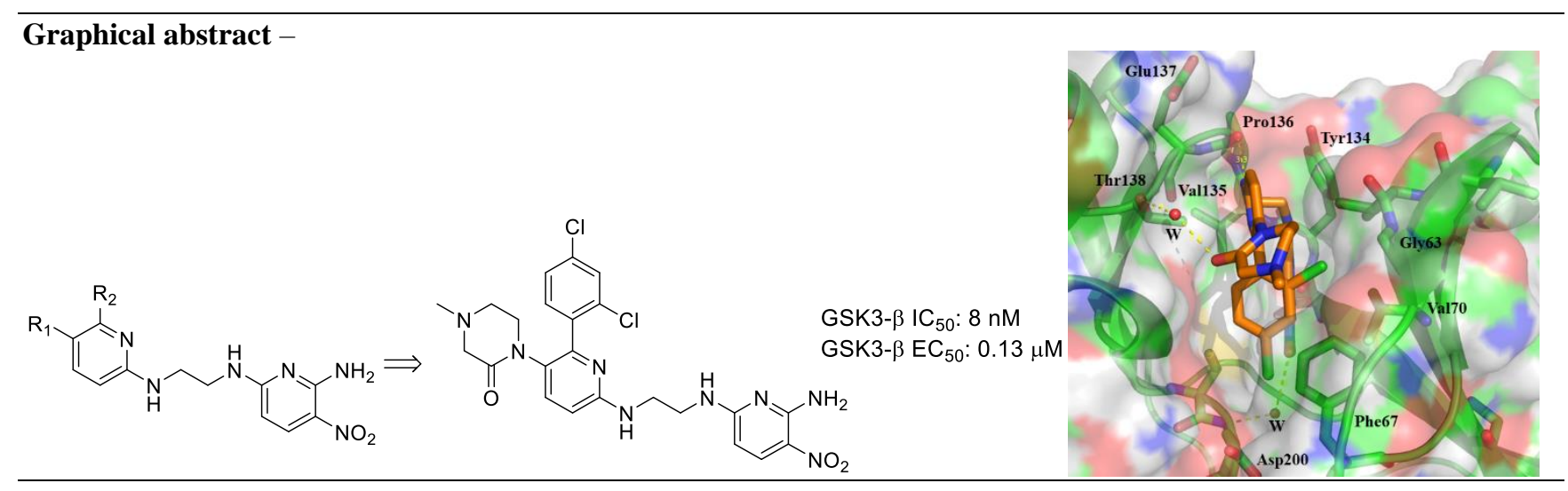


Keywords: GSK3- $\beta$, Diabetes, Structure-Based Drug Design

Abbreviations: DIEA, N,N-Diisopropylethylamine; GSK3, glycogen synthase kinase-3; HPLC, high-performance liquid chromatography; IR-CHO, insulin resistant Chinese hamster ovary cells; LCMS, liquid chromatography-mass spectrometry; NIDDM, non-insulin dependent diabetes mellitus; ZDF, Zucker diabetic fatty

*Corresponding author: Tel: (510) 879-9505; email: dirksen.bussiere@ @novartis.com

Non-insulin dependent diabetes mellitus (NIDDM), also known as type II diabetes is an increasingly prevalent disease in aging populations and is characterized by distinct metabolic changes, including a decreased sensitivity to insulin and a compensatory elevation in circulating insulin concentrations. Glycogen synthase kinase (GSK3) ${ }^{1-3}$ is one of many signaling components downstream from the insulin receptor (IR). GSK3 is a serine/threonine kinase for which 2 isoforms, $\alpha$ and $\beta$, have been identified ${ }^{4}$. Both isoforms are constitutively active in resting cells, but show distinct tissue distribution. GSK3 was originally identified as a kinase responsible for inactivating glycogen synthase (GS) by direct phosphorylation ${ }^{5,6}$. During insulin signaling, GSK3- $\beta$ is inactivated by a specific phosphorylation event near its $\mathrm{N}$-terminus, leading to increased GS activity and increased glucose disposal into glycogen. The central role of GSK- $\beta$ in glucose metabolism makes it an exciting target for controlling hyperglycemia, and inhibition of GSK3- $\beta$ represents a novel mechanism for improving glucose disposal in an insulin-conserving manner ${ }^{7,8}$.

GSK3 has been the focus of intensive medicinal chemistry efforts and many GSK3 inhibitors have been published $^{9-11}$. Our earlier publication presents a novel class of pyrimidines, which are both potent and exquisitely selective inhibitors of GSK3 $3^{12-14}$. In order to improve the physicochemical, pharmacokinetic and pharmacological properties of the pyrimidine series, a novel class of pyridines as was synthesized. Replacing the central pyrimidine ring in compound $\mathbf{1}$ (known as CHIR-911, CT-99021, or CHIR-73911; $\mathrm{EC}_{50}$ of 0.77 $\mu \mathrm{M}$ ) with a pyridine ring provided a new scaffold with improved cell permeability as shown by IR-CHO cell activity $\left(\mathrm{EC}_{50}\right.$ of $\left.0.13 \mu \mathrm{M}\right)$. The pharmacokinetic profile was also improved, showing longer half-life in both Zucker diabetic fatty (ZDF) rats (205 min) and beagle dogs (163 min) compared to CHIR-73911. We discovered that the metabolically labile imidazole in the pyrimidine core could be replaced with a monoketopiperazine (MKP) group in the pyridines. This resulted in a compound $\mathbf{2}$ (known as CHIR-637, CT-20026, or CHIR-118637) with improved solubility and stability over the earlier pyrimidine series.

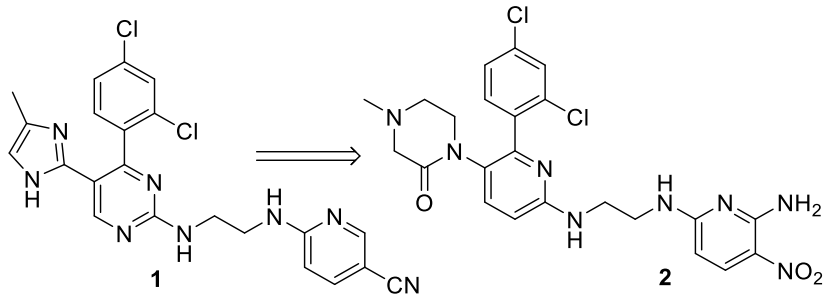

Two structural features were found to be critical in both series. Earlier studies showed that 2,4-dichlorophenyl was the optimal group at the 4-position, which forms a highly specific interaction with the P-loop of the kinase. Likewise, the ethylene diamine linker between the arenes was required for the GSK3 activity. The synthesis of the pyridines (Scheme 1) was initiated from the 2,6-dichloro-3-nitropyridine. Selective Suzuki coupling at the 2-position was achieved after some optimization. The 2-chloro group was selectively substituted when the 2,6-dichloronitro pyridine was reacted with the corresponding boronic acids, in the presence of triphenylphosphite-palladium complex with sodium carbonate in THF/Water (3:1) to provide 2-aryl substituted nitrochloropyridine. The ethylene diamine was introduced via SnAr with acetonitrile as solvent, in the presence of DIEA as base. The resulting nitro pyridine was reduced to the aniline and was acylated with the corresponding acylbromides. The acylbromide was then displaced with aminoethanol, which was in turn cyclized to give the MKP ring under Mitsunobu conditions to yield 2 . 

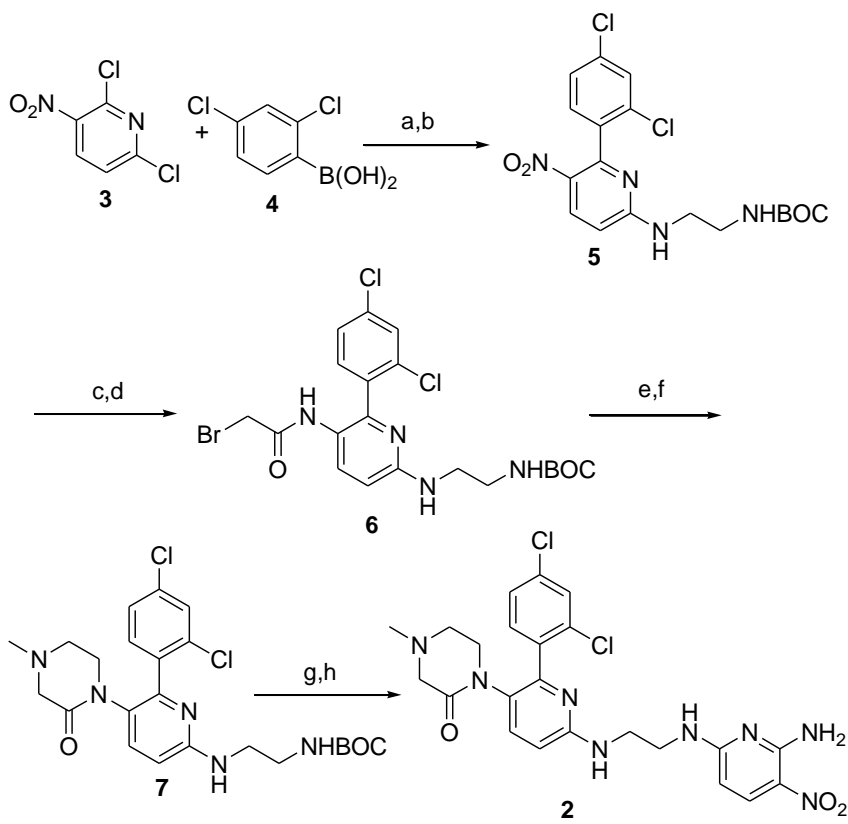

Scheme 1. General synthesis of pyridines, 7-14. Reagents and conditions: (a) $\mathrm{Pd}\left(\mathrm{Ph}_{3} \mathrm{P}\right)_{3}$, THF, water, rt; (b) $\mathrm{NH}_{2} \mathrm{CH}_{2} \mathrm{CH}_{2} \mathrm{NHBOC}$, DIPEA, $\mathrm{CH}_{3} \mathrm{CN}, \quad 90^{\circ} \mathrm{C}, 2 \mathrm{~h}$; (c) Fe, acetic acid, ethanol; (d) $\mathrm{BrCH}_{2} \mathrm{COCl}, \quad \mathrm{NaHCO}_{3}$, EtOAc, water, $0^{\circ} \mathrm{C}$ to $\mathrm{rt}$; (e) $\mathrm{CH}_{3} \mathrm{NHCH}_{2} \mathrm{CH}_{2} \mathrm{OH}, \mathrm{CH}_{3} \mathrm{CN}, 50^{\circ} \mathrm{C}, 2 \mathrm{~h}$; (f) $\mathrm{PH}_{3} \mathrm{P}, \mathrm{DIAD}, \mathrm{THF}, 70^{\circ} \mathrm{C}$, 16h; (g) 4N HCl, dioxane, methanol; (h) 6-chloro-3-nitropyridine-2amine, DIPEA, $\mathrm{CH}_{3} \mathrm{CN}, 90^{\circ} \mathrm{C}, 2 \mathrm{~h}$.

Extensive SAR data was generated for the pyridine series, and Table 1 lists the potency of the compounds with different aryl substitutions. Detailed assay protocols are given elsewhere ${ }^{15}$. These compounds were tested in both cell-free and cell-based assays. Groups such as 4-chlorophenyl (8), 2-chlorophenyl (9), 3-chlorophenyl (10), 2,4-difluorophenyl (11), 4ethylphenyl (12) and 2-trifluoromethylphenyl (13) were less active in comparison with its 2,4dichlorophenyl analog (2) in the cell free assay. The 4chlorophenyl (8) and 2-trifluoromethylphenyl (13) analogs were more active in an insulin-responsive $\mathrm{CHO}$ cell line. All $\mathrm{Ar}_{1}$ replacements for the 2,4dichlorophenyl group, including the unsubstituted phenyl (14) and heteroycyclic analog (15) proved less potent than the parent compound 2.
Table 1. GSK3 $\mathrm{IC}_{50}$ and $\mathrm{EC}_{50}$ assay results for compounds $\mathbf{2 , 8 - 1 5}$

\begin{tabular}{cccc} 
& & \\
& & \\
\hline Compounds & $\mathrm{Ar}_{1}$ & $\mathrm{IC}_{50}(\mathrm{nM})$ & $\mathrm{EC}_{50}(\mu \mathrm{M})$ \\
\hline $\mathbf{2}$ & 2,4-dichlorophenyl & 8 & 0.13 \\
$\mathbf{8}$ & 4-chlorophenyl & 44 & 0.76 \\
$\mathbf{9}$ & 2-chlorophenyl & 29 & 1.35 \\
$\mathbf{1 0}$ & 3-chlorophenyl & 74 & 3.00 \\
$\mathbf{1 1}$ & 2,4-diflurophenyl & 69 & 1.08 \\
$\mathbf{1 2}$ & 4-ethylphenyl & 52 & 0.29 \\
$\mathbf{1 3}$ & 2-trifluromethylphenyl & 57 & 0.76 \\
$\mathbf{1 4}$ & Phenyl & 202 & $\mathrm{ND}$ \\
$\mathbf{1 5}$ & 3-furyl & 3700 & $\mathrm{ND}$ \\
\hline
\end{tabular}

Varying the monoketopiperazine substituent in $\mathbf{2}$ generated additional SAR. The monoketopiperazine replacements given in Table 2 exhibited similar $\mathrm{IC}_{50}$ values in the cell-free GSK3 assay. Replacement of the methyl group in the MKP ring with a cyclopropyl ring, as in example 17 had very little effect on both the $\mathrm{IC}_{50}$ and $\mathrm{EC}_{50}$ values as compared to 2 . The removal of the charge on the nitrogen of the piperazinone ring was not tolerated as observed by the $\mathrm{EC}_{50}$ values. Compound 18, obtained by the acylation of the nitrogen on the piperazinone ring, and 19, obtained by the trifluroethylation of the nitrogen, showed lower $\mathrm{EC}_{50}$ values compared to 2 . Replacing the monoketopiperazine ring with a morpholine as in $\mathbf{2 0}$ exhibited a moderate $\mathrm{EC}_{50}$ value when compared to $\mathbf{2}$. The removal of the nitrogen to make a 5- or 6membered lactam, as in $\mathbf{2 1}$, was productive and maintained an equivalent $\mathrm{EC}_{50}$. Compound $\mathbf{2 2}$ was also productive. Compound 23, derived by the ring opening of the MKP, was not favorable as observed by the $\mathrm{EC}_{50}$ value. 
Table 2. GSK3 $\mathrm{IC}_{50}$ and $\mathrm{EC}_{50}$ assay results for compounds 16-23

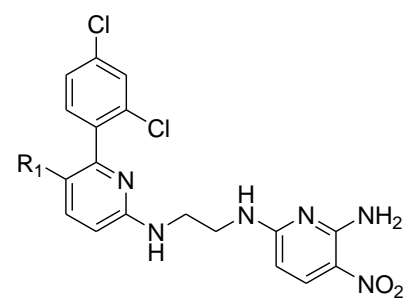

22 Compounds

Table 3 illustrates compounds in which the ethylene diamine linker was modified. The NH adjacent to the distal pyridine is essential as a hydrogen-bond donor to the hinge region, which is shown in the section that follows. At the proximal amine, compound 24 shows that substituting the nitrogen with methyl did not alter the compound's potency significantly. Likewise, the scaffold accommodates the introduction of a methyl group on the ethylene diamine linker. The S-isomer, 25, exhibited a minimal decrease in potency compared to the R-isomer, 26, which is 20-fold less potent.

Table 3. $\mathrm{GSK} \mathrm{IC}_{50}$ and $\mathrm{EC}_{50}$ assay results for compounds $\mathbf{2 4 - 2 8}$ with modification of the ethylene diamine linker

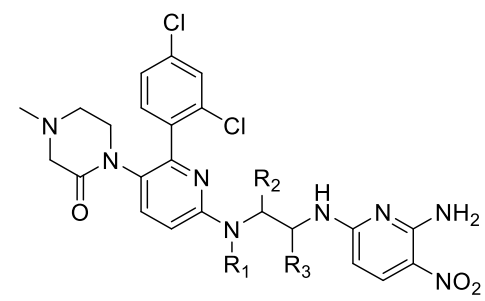

\begin{tabular}{cccccc}
\hline $\begin{array}{c}\text { Compou } \\
\text { nds }\end{array}$ & $\mathrm{R} 1$ & $\mathrm{R} 2$ & $\mathrm{R} 3$ & $\mathrm{IC}_{50}(\mathrm{nM})$ & $\mathrm{EC}_{50}(\mu \mathrm{M})$ \\
\hline $\mathbf{2 4}$ & $\mathrm{Me}$ & $\mathrm{H}$ & $\mathrm{H}$ & 15 & 0.22 \\
$\mathbf{2 5}$ & $\mathrm{H}$ & 'S'-Me & $\mathrm{H}$ & 36 & 0.31 \\
$\mathbf{2 6}$ & $\mathrm{H}$ & 'R'-Me & $\mathrm{H}$ & 207 & $\mathrm{ND}$ \\
$\mathbf{2 7}$ & $\mathrm{H}$ & $\mathrm{H}$ & 'S'-Me & 66 & 1.05 \\
$\mathbf{2 8}$ & $\mathrm{H}$ & $\mathrm{H}$ & 'R'-Me & 1 & $\mathrm{ND}$
\end{tabular}

To fully rationalize the observed SAR, we determined the co-structure of $\mathbf{2}$ bound to human GSK3- $\beta$ by $X$-ray crystallography. This was very challenging and requires a unique crystallization protocol ${ }^{15}$. The necessity to develop this protocol is likely due to the significant structural shifts that the kinase undergoes upon binding of the compound, which lead to the rapid disintegration of an apo-crystal upon soaking with compound. The compound series is ATPcompetitive, and, not surprisingly, occupies the volume of the kinase active site occupied by ATP. The interactions of $\mathbf{2}$ which lead to the exceptional potency against GSK3- $\beta$ are shown in Figure 1. There is a donor-acceptor-donor interaction between the 6-amino5-nitro-pyridine group and the 'hinge' region between the $\mathrm{N}$ - and $\mathrm{C}$-terminal domains of the kinase. The exocyclic amino group at the 2-position donates a hydrogen-bond to main-chain carbonyl of Val135 at the $\mathrm{n}+2$ position, the nitrogen of the pyridine ring accepts a hydrogen-bond from the main-chain amide of Val135, and the exocyclic amino group at the 6-position donates a hydrogen-bond to the main-chain carbonyl of Asp133. The nitro group at the 5-position accepts a hydrogen-bond from a structural water at the base of binding pocket; this structural water in turn donates a hydrogen-bond to the main-chain amide of Asp200. The relative arrangements of the amino groups and 
nitro group serve to polarize the proximal pyridine for optimal hydrogen-bonding to the hinge. The compound's ethylene diamine linker spans the binding site and positions the 2,4-dichlorophenyl group to form hydrophobic contacts by positioning the chloro groups both in a hydrophobic 'dimple' within the P-loop, formed by residues Ile62, Gly63, and Val70, and in a hydrophobic pocket at the bottom of the ATP binding pocket, formed by residues Val70, Lys85, and Asp200. This represents the optimal position for the chloro groups. A $\pi-\pi$ interaction is putatively formed between Phe67, the residue positioned at the tip of the P-loop, and the 2,4-dichlorophenyl group, which in turn forms a $\pi-\pi$ interaction with the nitroaminopyridine group. Formation of the $\pi-\pi$ interaction with Phe67 and the compound requires the phenylalanine to move more than $7 \AA$ from its position in the ATP-bound form. The 2,4-dichlorophenyl ring occupies the volume typically occupied by the ribose ring of ATP. The linker length and linker flexibility are critical components in positioning the hydrogen-bonding groups of the nitroaminopyridine as well as the 2,4-dichlorophenyl group. The monoketopiperazine group forms a hydrogen-bond with an adjacent structural water, which in turns forms a hydrogen-bond with the sidechain of Thr138. Finally, there is likely a weak hydrogen-bond formed between the ethylene diamine linker and the mainchain carbonyl of Pro136. The kinase maintains an active, DFG-in conformation with the compound bound (see Protein Data Bank accession code ----).

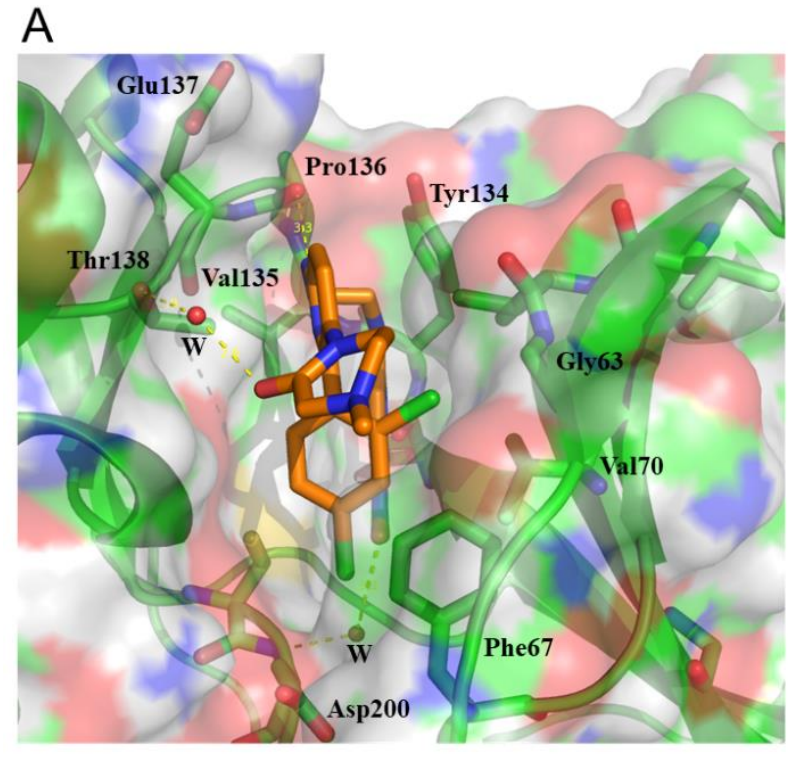

\section{B}

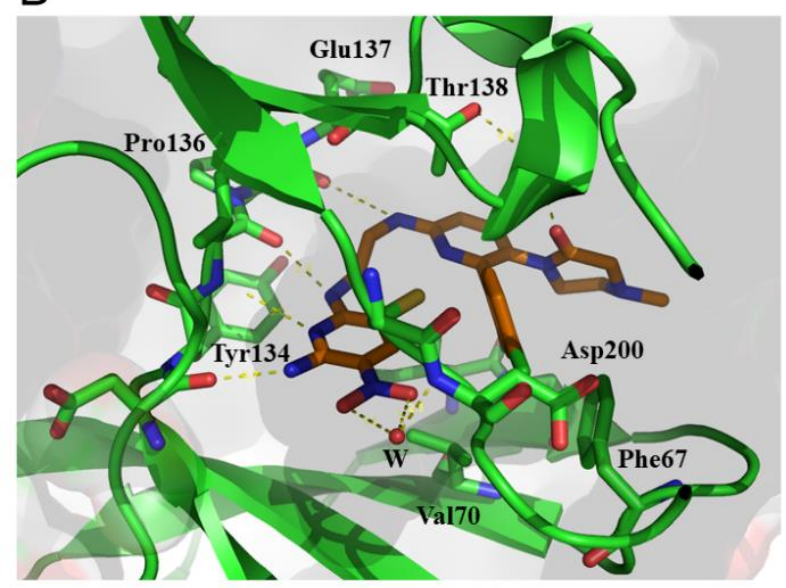

Figure 1. Co-crystal structure of 2 in the ATP-binding site of human GSK3- $\beta$. The resolution of the co-structure is approximately 2.6 Angstroms. The electrostatic character is superimposed on the solventaccessible surface of human GSK3- $\beta$. The visualizations were performed using the PYMOL program ${ }^{16}$. Red indicates negative potential, blue indicates positive potential, and green indicates a neutral (aliphatic) area. The N-terminal and C-terminal domains of the kinase are positioned as indicated. Key residues are labeled. Structural waters are labeled as 'W'. (A) Overall view of the ATP-binding pocket with 2 bound; key hydrogen bonds are shown as dashed lines and key residues are labelled; key waters are shown as red spheres; the $\mathrm{N}$-terminal $\beta$ strand kinase domain is to the right and the $\mathrm{C}$-terminal helical kinase domain is to the left. (B) Orthogonal view of the pocket at an approximately $90^{\circ}$ rotation along both axis; the $\mathrm{N}$-terminal $\beta$-strand kinase domain is on the top and the C-terminal helical kinase domain is on the bottom. The coordinates are available in the Protein Data Bank and can be accessed by PDB accession code ----.

The compounds in the pyridine series are extremely selective, showing from a hundred-fold to thousandfold selectivity against most human kinases as illustrated in the selectivity profile of $\mathbf{2}$ (Figure 2). Several interactions form the basis of the exquisite selectivity of $\mathbf{2}$. The first is the hydrogen-bonding of the compound with the main-chain carbonyl of Val135. 
The presence of Pro136 at the $n+3$ position ensures that the main-chain carbonyl faces inwards towards the binding site to allow this interaction. Only a few human kinases maintain a proline at the $n+3$ position of the hinge region ${ }^{17}$. The direct interaction of the compound linker with Pro136 also provides some selectivity for the same reasons. Finally, the positioning of the 2,4dichlorophenyl group allows the insertion a chlorogroup into the hydrophobic dimple within the P-loop and also allows the interaction between the 2,4dichlorophenyl group and Phe67 to form a face-to-face $\pi-\pi$ interaction. These two crucial interactions can be formed without compromising the donor-acceptordonor interactions formed with the hinge. In other closely related kinases, sequence differences within the P-loop that form the hydrophobic dimple or the residue in an equivalent position to Phe67 prevent the possibility for the formation of the hydrophobic dimple and/or the $\pi-\pi$ interaction at the tip of the P-loop. For example, in CDC2 kinase, where the sequence within the P-loop is highly homologous, there is a tyrosine (Tyr15) at the position equivalent to Phe67 at the tip of the P-loop. However, an equivalent conformation of the tyrosine sidechain which allows a similar $\pi-\pi$ interaction to form with the 2,4-dichlorophenyl group cannot be attained as the exocyclic hydroxyl group of the tyrosine group would collide with the main-chain of the P-loop. This collision prevents the necessary conformation and thus the formation of the interaction. So-called 'folded P-loop' conformations have been seen with other selective kinase inhibitors and are associated with a high-degree of selectivity ${ }^{18,19}$. As GSK3- $\alpha$ and $-\beta$ have virtually no sequence differences in the ATP-binding pocket, $\mathbf{2}$ is not isoform selective.

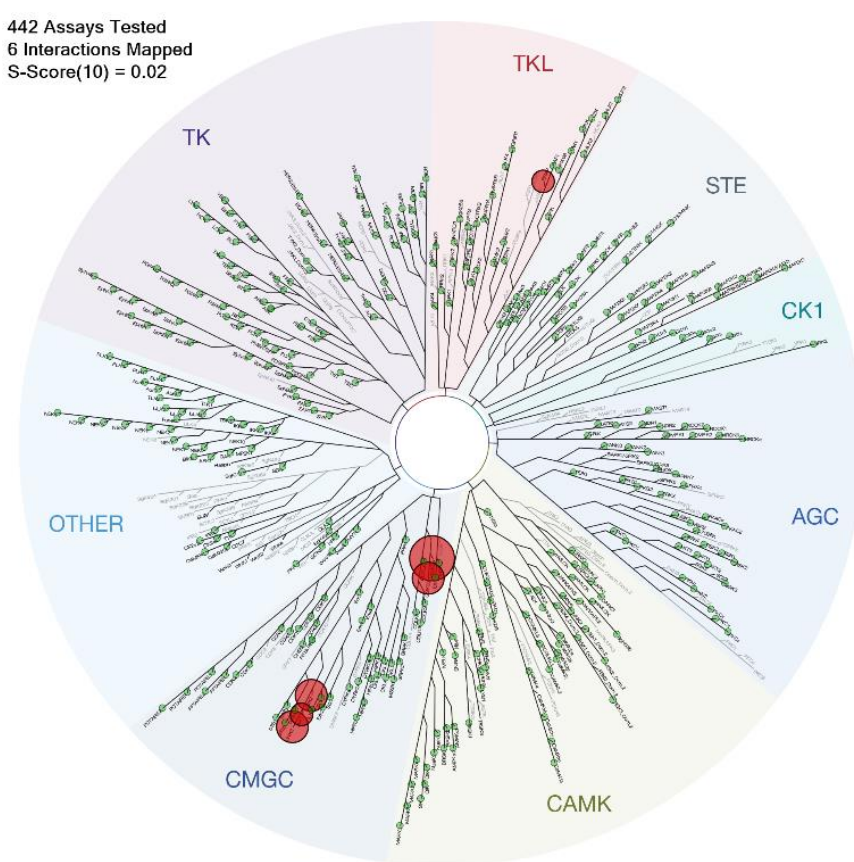

Figure 2. KINOMEscanTM profiling of 2. Kinase activities were screened at a concentration of $10,000 \mathrm{nM}(10 \mu \mathrm{M})$ of $\mathbf{2}$. Kinases whose activities were inhibited at levels of $90 \%$ or greater are denoted by red circles. The most potently inhibited kinases are: GSK3- $\alpha(99.45 \%$ inhibition), GSK3- $\beta$ (95.8\% inhibition), JNK1 ( $92.2 \%$ inhibition), JNK2 ( $97.4 \%$ inhibition), JNK3 (98.3\% inhibition), and B-RAF (91.8\% inhibition). The S-score (10) gives the fraction of the 442 kinases tested that were inhibited at $90 \%$ of baseline or greater. Image generated using TREEspot $t^{\mathrm{TM}}$ Software Tool and reprinted with permission from

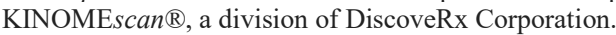

In addition, the use of a 2-aminopyridine core in place of a pyrimidine core generally yielded compounds with a better overall metabolic profile and improved cell permeability and longer half-life $\left(\mathrm{t}_{1 / 2}\right)$ in ZDF rats $(205$ min versus $92 \mathrm{~min}$, for $\mathbf{2}$ and $\mathbf{1}$, respectively). In beagle dogs, the half-life was 163 min. In an oral Glucose tolerance test (oGTT) in ZDF rats, the compound exhibited an $\mathrm{ED}_{50}$ of $10 \mathrm{mg} / \mathrm{kg}$ after a single oral administration with a $30 \%$ oral bioavailability.

In conclusion, a series of potent and selective GSK3 inhibitors were derived from 5-nitro- $\mathrm{N}^{2}$-(2-(pyridine2ylamino)ethyl)pyridine-2,6-diamines. Exploration of the scaffold's structure/activity relationships and pharmacology revealed that $\mathbf{2}$ had superior in vitro and in vivo properties than the previously described pyrimidine series ${ }^{15}$. In addition to their demonstrated efficacy in the treatment of NIDDM, the compounds have been investigated for use in maintaining embryonic stem cells in a pluripotent state for efficient culturing ${ }^{20,21}$, as well as for the treatment of hair loss and hair follicle regeneration ${ }^{22}$. Recent work in natural products has suggested that these compounds and other GSK3 inhibitors could serve as an entry point for the treatment of epilepsy ${ }^{23}$. Complete synthetic procedures for presented compounds are available ${ }^{24}$. 


\section{Author contributions}

All authors have given approval to the final version of this manuscript.

\section{Acknowledgements}

We thank Weiping Jia for mass spectrometry services, Alice Wang for compound purification and Anu Sharma for collecting HPLC and LCMS data for several compounds. We thank Drs. Lewis Williams, Yumi Nakagawa, and Jacob Plattner for their advice and mentorship during the project. This research was funded by Chiron Corporation and the Novartis Institutes for BioMedical Research.

\section{References}

1. Woodgett JR, Cohen P. Multisite phosphorylation of glycogen synthase. Molecular basis for the substrate specificity of glycogen synthase kinase-3 and casein kinaseII (glycogen synthase kinase-5). Biochim Biophys Acta. 1984;788(3):339-347. https://doi.org/10.1016/0167-4838(84)90047-5

2. Eldar-Finkelman H, Ilouz R. Challenges and opportunities with glycogen synthase kinase-3 inhibitors for insulin resistance and Type 2 diabetes treatment. Expert Opin Investig Drugs. 2003;12(9):1511-1519. https://doi.org/10.1517/13543784.12.9.1511

3. Eldar-Finkelman H. Glycogen synthase kinase 3: an emerging therapeutic target. Trends Mol Med. 2002;8(3):126-132. https://doi.org/10.1016/S1471-4914(01)022663

4. Woodgett JR. A common denominator linking glycogen metabolism, nuclear oncogenes and development. Trends Biochem Sci. $1991 ; 16(5): 177-181$. https://doi.org/10.1016/0968-0004(91)90071-3

5. Grimes CA, Jope RS. The multifaceted roles of glycogen synthase kinase 3beta in cellular signaling. Prog Neurobiol. 2001;65(4):391426.

https://doi.org/10.1016/S0301-0082(01)000119

6. Cross DA, Alessi DR, Vandenheede JR, McDowell HE, Hundal HS, Cohen P. The inhibition of glycogen synthase kinase-3 by insulin or insulin-like growth factor 1 in the rat skeletal muscle cell line L6 is blocked by wortmannin, but not by rapamycin: evidence that wortmannin blocks activation of the mitogen-activated protein kinase pathway in L6 cells between Ras and Raf. Biochem J. 1994;303 ( Pt 1):21-26.

https://doi.org/ 10.1042/bj3030021

7. Nikoulina SE, Ciaraldi TP, Mudaliar S, Mohideen P, Carter L, Henry RR. Potential role of glycogen synthase kinase-3 in skeletal muscle insulin resistance of type 2 diabetes. Diabetes. 2000;49(2):263-271.

https://doi.org/10.2337/diabetes.49.2.263

8. DeFronzo RA, Bonadonna RC, Ferrannini E. Pathogenesis of NIDDM. A balanced overview. Diabetes Care. 1992;15(3):318-368. https://doi.org/10.2337/diacare.15.3.318

9. Kuo G-H, Prouty C, DeAngelis A, et al. Synthesis and Discovery of Macrocyclic Polyoxygenated Bis-7-azaindolylmaleimides as a Novel Series of Potent and Highly Selective Glycogen Synthase Kinase-3 $\beta$ Inhibitors. J Med Chem. 2003;46(19):4021-4031.

https://doi.org/10.1021/jm030115o

10. Olesen PH, Sørensen AR, Urs $\varnothing$ B, et al. Synthesis and in vitro characterization of 1-(4aminofurazan-3-yl)-5-dialkylaminomethyl-1H$[1,2,3]$ triazole-4-carboxylic acid derivatives. A new class of selective GSK-3 inhibitors. J Med Chem. 2003;46(15):3333-3341.

https://doi.org/10.1021/jm021095d

11. Witherington J, Bordas V, Garland SL, et al. 5aryl-pyrazolo[3,4-b]pyridines: potent inhibitors of glycogen synthase kinase-3 (GSK-3). Bioorg Med Chem Lett. 2003;13(9):1577-1580. https://doi.org/10.1016/S0960894X(03)00134-3

12. Wagman AS, Johnson KW, Bussiere DE. Discovery and development of GSK3 inhibitors for the treatment of type 2 diabetes. Curr Pharm Des. 2004;10(10):1105-1137. https://doi.org/10.2174/1381612043452668

13. Nuss JM, Harrison SD, Ring DB, Boyce RS, Johnson, K, Pfister, KB et al. Preparation of aminopyrimidines and -pyridines as glycogen synthase kinase 3 inhibitors. European patent application WO 0220495 A2 20020314. 2002; pp. 262. 
14. Ring DB, Johnson KW, Henriksen EJ, et al. Selective glycogen synthase kinase 3 inhibitors potentiate insulin activation of glucose transport and utilization in vitro and in vivo. Diabetes. 2003;52(3):588-595.

https://doi.org/10.2337/diabetes.52.3.588

15. Wagman AS, Boyce RS, Brown SP, et al. Synthesis, Binding Mode, and Antihyperglycemic Activity of Potent and Selective (5-Imidazol-2-yl-4-phenylpyrimidin2-yl)[2-(2-pyridylamino)ethyl]amine Inhibitors of Glycogen Synthase Kinase 3. J Med Chem. 2017;60(20):8482-8514.

https://doi.org/10.1021/acs.jmedchem.7b00922

16. DeLano WL. Pymol: An open-source molecular graphics tool. CCP4 Newsletter on Protein Crystallography, 2002; 40: 92-92.

17. Bullock AN, Russo S, Amos A, et al. Crystal structure of the PIM2 kinase in complex with an organoruthenium inhibitor. PLoS One. 2009;4(10):e7112.

https://doi.org/10.1371/journal.pone.0007112

18. Guimarães CRW, Rai BK, Munchhof MJ, et al. Understanding the impact of the P-loop conformation on kinase selectivity. J Chem Inf Model. 2011;51(6):1199-1204.

https://doi.org/10.1021/ci200153c

19. Müller S, Chaikuad A, Gray NS, Knapp S. The ins and outs of selective kinase inhibitor development. Nat Chem Biol. 2015;11(11):818821.

https://doi.org/10.1038/nchembio.1938

20. Mays RW. Culture of Non-Embryonic Cells at High Cell Density. US patent application 2008/0194024 A1. 2008; pp. 31.

21. Perry JM, Li L, Grindley, JC. Methods and compositions for stem cell self-renewal. US patent 9,771,559. 2017; pp. 47.

22. Alvarez A. Apparatus and method for tissue rejuvenation. US patent application 2014/0276362A1. 2014; pp. 26.

23. Aourz N, Serruys A-SK, Chabwine JN, et al. Identification of GSK-3 as a Potential Therapeutic Entry Point for Epilepsy. ACS Chem Neurosci. 2019; 10(4): 1992-2003. https://doi.org/10.1021/acschemneuro.8b00281
24. Nuss JM, Harrison SD, Ring D.B., Boyce RS, Brown SP, Goff DA et al. Inhibitors of glycogen synthase kinase 3. United States patent 7,037,918. 2006; pp. 118. 